\title{
Research Digest
}

\section{Synopses of Research Articles}

\section{On the Brink: How Biology and Humans Affect Extinction Risk}

Close to a quarter of the world's mammals are at high risk of extinction. Save for the periodic "great extinctions," mammalian extinction has been a relatively rare event in geological terms, with one species disappearing from the fossil record every 1,000 years or so. Over the past 400 years, species have been disappearing 50 times faster than this "background" rate, with one vanishing every sixteen years. Human population growth and all its consequences-habitat destruction, propagation of invasive species, poaching - are largely to blame. Top predators often suffer heavily from encounters with humans, especially when those predators are perceived as economic threats. Thirty-four Mexican gray wolves have been reintroduced in Arizona since 1998 , and five have been shot, reportedly by ranchers.

Species in the most densely populated areas are expected to face the greatest risk, yet some survive while others perish, suggesting biological factors play a role in their fate. If, for example, the same external force drastically reduces populations of species with different biological profiles, then a species with a relatively short gestation period may stand a better chance of recovering than a long-gestating species.

Effective conservation strategies depend on understanding which factors are likely to increase extinction risk, but it's unclear how important intrinsic biological traits are relative to external pressures from humans and whether biology's influence on survival depends on the intensity of the threat. Ecologists often use human population density as a proxy for anthropogenic threats such as habitat destruction and hunting. To tease out the relative importance of all these factors, Marcel Cardillo et al. analyzed the impact of various biological traits and human population density on extinction risk in the mammal order Carnivora, which includes the red panda, lion, and members of the photogenic weasel-like viverrid family. By identifying the most salient factors in predicting extinction, the authors have created a model to identify those species at greatest risk.

The biology of a species combined with human population density, the researchers found, is a stronger predictor of risk than exposure to humans alone; those biological traits that increase risk vary depending on a species' exposure to human populations. Carnivores with low exposure to humans, for example, are likely to be at greater risk if their population density is low and they have small ranges, possibly because this makes them more vulnerable to loss of habitat. Species living near densely populated human areas must often contend with hunting and other direct threats on top of habitat loss and are more at risk if they also have long gestation periods - they can't repopulate fast enough to offset the additional pressures. Based on projected human population growth, this model predicts the addition of a number of species-mostly from Africa, where population growth rates largely exceed the global average-to the endangered list by the year 2030. Most of these species-including African viverrids such as the common genet, which not only lives in areas where human populations are rapidly expanding but is also biologically predisposed to decline-are currently considered a low conservation priority.

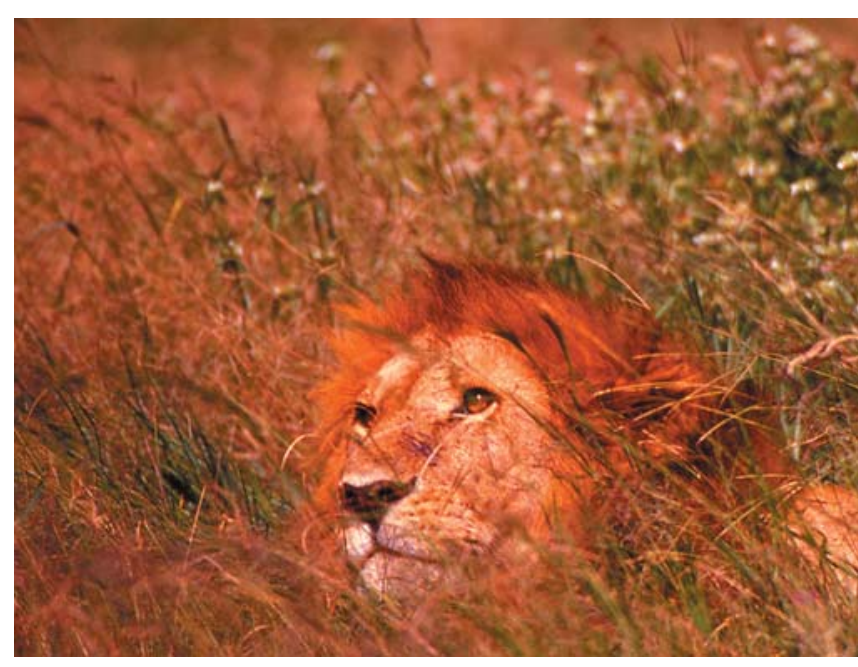

The IUCN lists lions as vulnerable. Photo, with permission, by Nicky Jenner, Institute of Zoology, London.

While it's possible that the direct effects of human population density are past - that is, species most sensitive to human incursions are already gone-human population density likely modulates biology. That might explain why gestation length didn't predict risk for species living in sparsely populated areasall else being equal, their numbers remained relatively stable. $A$ species with a small population forebodes a high extinction risk regardless of human density, though species with long gestation periods, again, face greater danger in the company of humans.

Altogether, these results suggest that as human population pressures increase, the importance of species-specific biology in predicting extinction risk also increases, with biology affecting which species are most vulnerable to external threats. With most conservation efforts focused on damage control, these findings make the case for interceding before a species reaches the brink of extinction. "There is no room for complacency about the security of species," the authors warn, "simply because they are not currently considered threatened."

Cardillo M, Purvis A, Sechrest W, Gittleman JL, Bielby J, et al. (2004) Human population density and extinction risk in the world's carnivores. DOI: 10.1371/journal.pbio.0020197

\section{Honeybees' Distance Perception Changes with Terrain of Flight Path}

When a trip for food can require a three-mile flight, it pays to get the directions right, especially if you're a bee. Bees more typically forage within a 600- to 800-yard radius, expending a significant amount of energy - a fact they seem keenly aware of: for reasons that remain unclear, bees tend to ignore directions that send them to a target on water. It's been known since Aristotle's time that returning foragers dance a little jig for their hivemates, presumably regaling them with tales of nectar-laden flora. Some 2,300 years later, zoologist Karl von Frisch correlated dance choreography to the direction and distance of a food source, eventually winning the Nobel prize for his work. Since then, researchers have been working out the details of bee 
communication, such as how fellow foragers interpret the "waggle dance" and how dancers perceive and convey navigational details of a trip. A key aspect of this information exchange is how bees estimate distance.

Recent studies suggest that bee odometers are driven by the "optic flow" experienced during flight_-or, simply put, bees appear to log distance by measuring the rate that images of passing terrain move in their eye during flight. This theory comes from observations that when bees fly a given distance, they indicate a much longer distance-by performing a longer waggle-for low-flying trips than for those at higher altitudes, presumably because flying at higher altitudes limits the bees' ability to perceive changing images. Likewise,

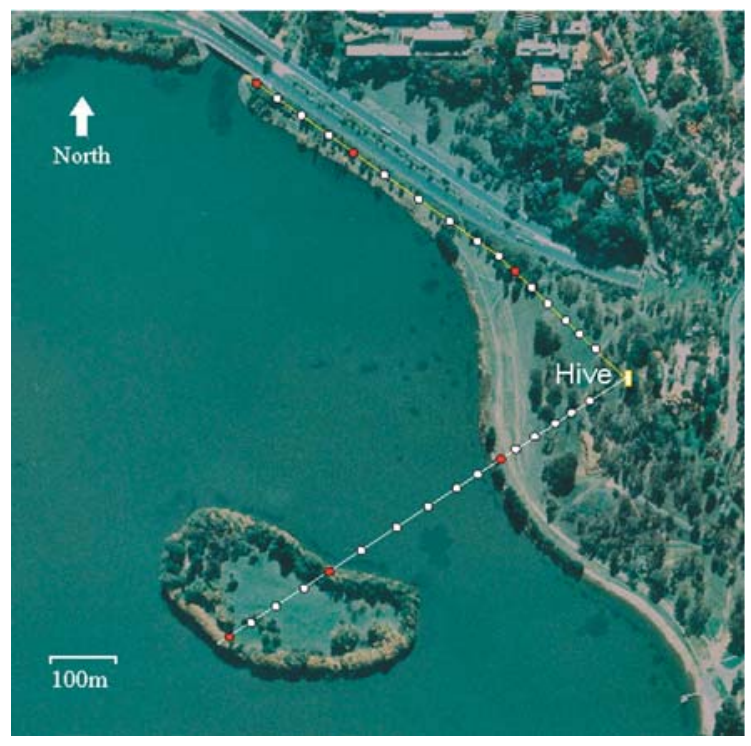

A honeybee's "odometer" generally runs faster when it flies over land than water

bees flying through short, narrow tunnels filled with visual elements waggle a disproportionately long distance. These observations also raise questions about which visual aspects of the environmentcontrast, texture, distribution of objectsare most important to a bee's perception of image flow.

To investigate the factors driving the bee trip odometer, Mandyam Srinivasan and colleagues trained bees to feed at locations along two different routes in a natural environment, then compared their waggle dances. One route was entirely over land; the other started on land, shifted to water, and ended back on land. Both routes were the same distance, about 630 yards. Bees trained to feed at a boat in the middle of a lake had no trouble getting there, which was no guarantee based on reports that bees have trouble flying across lakes, often plunging into the drink. They had less success recruiting their colleagues to share in the bounty, even though their waggles clearly placed the feeder on the lake. (This finding supports an earlier, controversial theory that suggests experienced bees know water rarely harbors bee food.)

The length of the bees' waggle dance increased faster with distance when they flew over land than when they flew over water. This disparity indicates that land provides a stronger "odometric signal" than water."The honeybee's odometer," the authors explain,"runs at a slower pace when flight is over water." Overland flights tend to offer high contrasts and rich textures, while flights over water tend to offer low contrasts and sparse textures. Most likely, it is the high contrast of land surfaces that triggers a stronger odometric signal. But land surfaces also show variation in contrast, which was reflected in the bees' dance. One section of the land-only route was a paved bicycle path, a low contrast surface that the bees waggled as a relatively shorter distance.

Whether or not the contrast theory holds, Srinivasan and colleagues conclude, differences in the visual environment trigger differences in odometric signal. The odometer racks up yards depending on the nature of the terrain, whether it be land or water, during flight. The great Belgian playwright and avid bee-keeper Maurice Maeterlinck wondered at the language of bees in his 1901 book, The Life of the Bee, deciding it must correspond "to senses and properties of matter wholly unknown to ourselves." As Srinivasan and colleagues show here, the bee's view of the world indeed corresponds to a unique way of interpreting the landscape-and of sharing news of their travels with their hivemates.

Tautz J, Zhang S, Spaethe1 J, Brockmann A, Si A, et al. (2004) Honeybee odometry: Performance in varying natural terrain. DOI: 10.1371/journal.pbio.0020211
No Rest for the Weary: Migrating Songbirds Keep Their Wits without Sleep

Every spring and fall, billions of songbirds fly thousands of miles between their summer breeding grounds in North America and their wintering grounds in the more hospitable climes of southern California Mexico, and Central and South America. While some birds fly during the day, most, including the white-crowned sparrow, fly under cover of night. Many aspects of this remarkable voyage remain obscure, especially if, and how, nocturnal migrators get any sleep at night.

A tracking study of the Swainson's thrush found that the roughly seveninch birds flew up to seven hours straight on six of seven nights, racking up over 930 miles. While the study didn't track their daytime behavior, the birds' migratory pace-as well as the increased activity required to sustain migrations - suggests little time for sleep. Yet field observations indicate that presumably sleep-deprived fliers appear no worse for wear, foraging, navigating, and avoiding predators with aplomb. Researchers are left trying to reconcile this observation with the vast body of evidence linking sleep deprivation to impaired neurobehavioral and physiological function. How do songbirds cope with so little sleep? Do they take power naps? Have they taken "sleep walking" to new heights? Or have they managed to selectively short-circuit the adverse effects of sleep deprivation during migratory stints?

To investigate these questions, Ruth Benca and colleagues studied cognitive and sleep behaviors in captive whitecrowned sparrows over the course of a year. The sparrows fly nearly 2,700 miles twice a year between their Alaska and southern California homes. In laboratory cages, the birds' migratory instincts manifest as increased restlessness at night during the migratory season, with lots of hopping around and wing flapping.

Niels Rattenborg et al. characterized the birds' activity levels with motiondetection measurements and video recordings, and placed sensors on their brains to monitor their seasonal sleep patterns. The brain recordings 


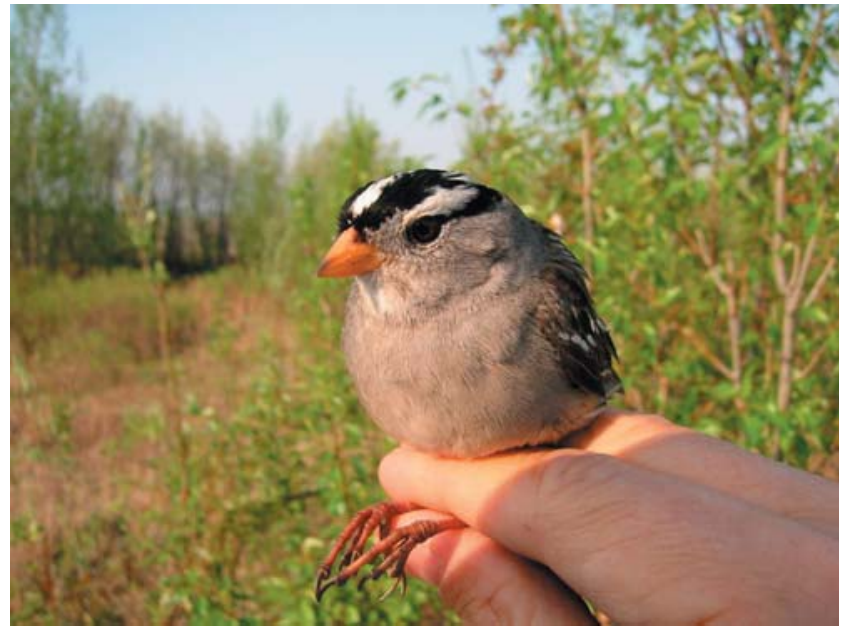

White-crowned sparrow (Zonotrichia leucophrys gambelii)

showed a marked seasonal difference in both the amount and type of sleep during a 24-hour period: migrating birds spent roughly two-thirds less time sleeping than nonmigratory birds and fell into REM sleep (the dream stage of sleep, marked by rapid eye movements) much sooner. Birds displaying active migratory behavior appeared completely awake during such activity. Cognitive tests-birds performed a task that involved pecking a key in exchange for seed-revealed that birds in the nonmigrating state suffered cognitive deficits when sleepdeprived but displayed an "unprecedented" ability to maintain cognitive function in the face of ongoing sleep loss in the migratory state.

These results suggest that wild songbirds drastically reduce sleep time during migration, though Benca and colleagues concede it's impossible to know for sure without recording the birds in action. And it is unclear what molecular mechanisms jumpstart the migratory mindset. Such an ability to temporarily circumvent the need for sleep, however, could prove useful for humans in situations that demand continuous performance. Some studies link migration with increased neuroendocrine activity, which is in turn associated with sleep disruption, accelerated timing of REM cycles, and mood disorders in humans. "Like migrating sparrows," the authors note, "both depressed and manic patients show reduced latency to REM sleep, loss of slow-wave sleep, and reduced amounts of total sleep." Given the parallels between migratory behaviors and bipolar illness, it's possible that similar mechanisms may be involved in both.

Whatever the mechanism, the unprecedented imperviousness of migrating songbirds to sleep deprivation, the authors conclude, clearly warrants further testing. But it also raises interesting questions about the role of sleep, which recent studies suggest is required to incorporate novel perceptions into the brain's memory banks. If this is true, how do songbirds consolidate memories of migratory events with so little sleep? Understanding the mechanisms that power the sleepless flight of songbirds promises to unravel one of the longstanding mysteries of their improbable journey. It may also shed light on the origins of sleep-related seasonal disorders and the muchdebated role of sleep itself.

Rattenborg NC, Mandt BH, Obermeyer WH, Winsauer PJ, Huber R, et al. (2004) Migratory sleeplessness in the white-crowned sparrow (Zonotrichia leucophrys gambelii). DOI: 10.1371/journal.pbio.0020212

\section{Great Ape Genomes Offer Insight into Human Evolution}

Some primatologists have argued that to understand human nature we must understand the behavior of apes. In the social interactions and organization of modern primates, the theory goes, we can see the evolutionary roots of our own social relationships. In the genomic era, the age-old question, What makes us human? has become, Why are we not apes? As scientists become more adept at extracting biological meaning from an ever expanding repository of sequenced genomes, it is likely that our next of kin will again hold promising clues to our own identity.

Many comparative genomics studies have looked to our more distant evolutionary relatives, such as the mouse and even yeast, to help interpret the human genome. Because the genomes of mice, yeast, and humans have diverged significantly since their last common ancestor-about 75 million years ago for mouse and human, and about 1 billion years ago for yeast and human-there are enough differences between the functional and nonfunctional regions to home in on biologically significant sequences, based on their similarity. Sequences that are similar, or conserved, in such divergent species are assumed to encode important biological functions. These comparative studies have successfully identified and characterized many human genes. And a similar approach comparing primate genomes can help scientists understand the genetic basis of the physical and biochemical traits that distinguish primate species. In this approach, however, rather than looking for genes that are shared across many species, scientists look for those that are unique to a species.

One of the primary agents of genome evolution is gene duplication. Duplicated genes provide the raw material for the generation of novel genes and biological functions, which in turn allow the evolution of organismal complexity and new species. (For more on duplicated genes, see the primer by Hurles in this issue.) James Sikela and colleagues set out to compare gene duplications between humans and four of our closest primate relatives to find the genetic roots of our evolutionary split from the other great apes. Collecting the DNA of humans, chimpanzees, bonobos, gorillas, and orangutans from blood and experimental cell lines, the researchers used microarray analysis to identify variations in the number of copies of individual genes among the different species. They analyzed nearly 30,000 human genes and compared their copy numbers in the genomes of humans and the four great apes.

Overall, Sikela and colleagues found more than 1,000 genes with lineage-specific changes in copy number, representing $3.4 \%$ of the genes tested. All the great ape species showed more increases than decreases in gene copy numbers, but relative to the evolutionary age of each lineage, humans showed the highest number of genes with increased copy numbers, at 134. Many of these duplicated human genes are implicated in brain structure and function.

The gene changes identified in the study, the authors conclude, likely represent most of the major lineage-specific gene expansions (or losses) that have taken place since orangutans split from the other great apes, some 15 million years ago. (Humans diverged from their closest cousins, the chimp and bonobo, roughly 5 million to 7 million years ago.) And because some of these gene changes were unique to each 


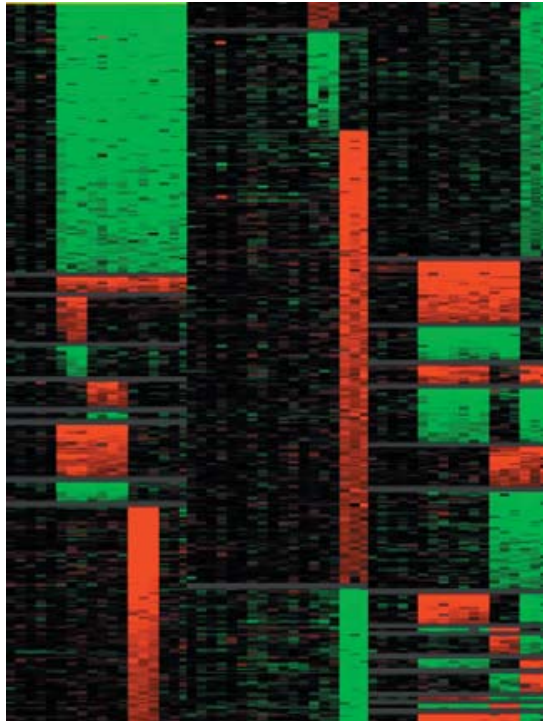

Lineage-specific gene gains and losses in humans and great apes

of the species examined, they will likely account for some of the physiological and morphological characteristics that are unique to each species. One cluster of genes that amplified only in humans was mapped to a genomic area that appears prone to instability in human, chimp, bonobo, and gorilla. This region, which corresponds to an ancestral region in the orangutan genome, has undergone modifications in each of the other descendent primate species, suggesting an evolutionary role. In humans, gene mutations in this region are also associated with the inherited disorder spinal muscular atrophy. This fact, along with the observation that there are human-specific gene duplications in this region, suggests a link between genome instability, disease processes, and evolutionary adaptation.

In their genome-wide hunt for gene duplications and losses in humans and great apes, Sikela and colleagues have highlighted genomic regions likely to have influenced primate evolution. With the impending release of the chimp genome and more primate sequences to follow, scientists can take advantage of both sequence-based and microarraybased genome information to wrest additional insights from our primate cousins and flesh out the details of the human story.

Fortna A, Kim Y, MacLaren E, Marshall K, Hahn G, et al. (2004) Lineage-specific gene duplication and loss in human and great ape evolution. DOI: 10.1371 /journal. pbio.0020207

\section{Regulating the Regulators: Immune System Regulators Are Highly Susceptible to HIV Infection}

Nearly a dozen varieties of interdependent cells work in harmony to protect the body from infectious pathogens. Dendritic cells and B-cells carry remnants of pathogens to nearby helper T-cells (also known as CD4 cells because they express the CD4 protein on their surfaces), which coordinate a threat response by signaling killer T-cells to destroy the intruder. Yet another class of cells cleans up the debris such an encounter inevitably creates. Each of these cell types is further classified based on the combinations of surface proteins they express. Such proteins may simply identify the cell type or may help define its function.

An immune response can activate millions of cells; once these cells perform their respective jobs, it's crucial that they retire from active duty. That's where regulatory T-cells, or Treg cells, come in: an immune system that fails to retreat from an immune response can be just as dangerous as one that fails to organize.

Research has recently focused on a subset of Treg cells that express CD4 and CD25 proteins (so-called CD $4{ }^{+} \mathrm{CD} 25^{+}$ T-cells) and that suppress T-cell activation in mice and humans. Treg cells appear to be key immune system regulators, as their absence results in autoimmune and allergic diseases. Now a team led by Derya Unutmaz at Vanderbilt University report that human Treg cells are highly vulnerable to HIV infection.

Treg cells isolated from healthy volunteers were not only highly susceptible to HIV infection but were also killed by the virus. Because Treg cells account for only about $1 \%$ of human T-cells and are difficult to grow in a test tube, Unutmaz and colleagues developed a way to manufacture sufficient quantities for study by introducing a transcription factor called FoxP3 into conventional "naïve" T-cells (cells not yet primed to recognize a specific target). FOXP3 is required for the development of Treg cells in mice. Though it's not clear what role the human form of the gene plays in human Treg cell development, FOXP3 mutations cause an autoimmune disease associated with hyperactive T-cells. Here the authors demonstrate that FoxP3 transforms T-cells into Treg cells. The FoxP3-engineered T-cells behaved just like naturally occurring Treg cells: when exposed to a population of resting naïve CD4 T-cells, the engineered cells suppressed their activation. Overexpression of FOXP3 also made activated T-cells more susceptible to infection.

Since Treg cells are so susceptible to HIV, the researchers reasoned that these cells might be compromised in HIV-infected patients and that loss of Treg cells could lead to T-cell hyperactivation. Their logic was borne out by the finding that a portion of HIV patients with low CD4 counts and high levels of activated T-cells also had greatly depleted numbers of FoxP3-expressing CD4 ${ }^{+} \mathrm{CD} 25^{+} \mathrm{T}$-cells-a marker for Treg cells. The finding that HIV targets cells that normally suppress immune function is significant, given that HIV infection is characterized by chronic T-cell hyperactivation. Disruption of Treg cells, Unutmaz and colleagues conclude, could in turn disrupt the delicate balance of immune system function, setting the stage for hyperactivation-and a chronically hyperactive immune response could eventually exhaust the immune system. With a method to generate large numbers of Treg cells for study, Unutmaz and colleagues have paved the way for identifying mechanisms that mediate Treg cells' suppressive function and provided another resource for determining how HIV tips the scales toward disease progression.

Oswald-Richter K, Grill SM, Shariat N, Leelawong M, Sundrud MS, et al. (2004) HIV infection of naturally occurring and genetically reprogrammed human regulatory T-cells. DOI: 10.1371/ journal.pbio.0020198 
chickens use gene conversion through adolescence, when the cells move from a hindgut organ called the

Viruses, bacteria, and other pathogens betray their presence in the body through exterior proteins, distinct to each strain.

To prepare for the multitude of potential infectious agents, developing B-cells shuffle their genes to produce as many as a billion different antibodies, one to match almost any foreign protein. Upon infection, a limited subset of these antibodies will recognize a particular pathogen and mobilize a larger, targeted immune response. B-cells producing the "recognizing" antibody refine and test genetic modifications, adjusting the antibody's fit to the foreign entity. B-cells compete for the best match, or highest affinity; the winners survive to produce more cells and more antibodies against the invader.

B-cells require an enzyme called activation-induced cytidine deaminase (AID) to develop the most effective antibody. AID generates mutations in the highly variable targetrecognition region of an antibody. Removing the AID gene prevents antibody refinement in mature human and mouse B-cells-which use a process called somatic hypermutation to alter single nucleotides in the antibody gene-as well as chicken cells that use a different process called gene conversion to produce variation. Unlike the single nucleotide changes caused by hypermutation, gene conversion modifies an antibody by swapping part of its antigen-binding region for a replacement gene segment. Preference for hypermutation versus gene conversion varies across species, and can even vary within a species. B-cells in bursa into the spleen, where hypermutation takes over. It is unclear precisely how AID induces either somatic hypermutation or gene conversion, and how it chooses one over the other. Several recent studies suggest that AID's effectiveness may depend on damage to a single DNA base-specifically, changing a cytidine to uracil, which AID can do in either DNA or RNA.

To test whether AID causes hypermutation and gene conversion through a common pathway, Jean-Marie Buerstedde and colleagues at the National Research Center for Environment and Health in Munich, Germany, deleted the donor genes that supply replacement segments for gene conversion in chicken bursa cells. The cells not only stopped performing gene conversion; they revved up single nucleotide mutations in a pattern that looked suspiciously like somatic hypermutation. The mutations targeted hotspots for gene conversion, suggesting that hypermutation and gene conversion share common starting points along Occurrence of Ig gene conversion and hypermutation on an evolutionary tree antibody genes. This paper adds evidence that AID functions by swapping a single DNA base to induce multiple modes of gene shuffling and refinement in B-cells.

Arakawa H, Saribasak H, Buerstedde J-M (2004) Activation-Induced Cytidine Deaminase initiates immunoglobulin gene conversion and hypermutation by a common intermediate. DOI: 10.1371 /journal. pbio.0020179

\section{Calling the Steps in Development's Genetic Square Dance}

A single, fertilized egg divides into apparently identical daughter cells. As these twins divide again and again, differences emerge among their progeny, establishing segments that will distinguish back from front and head from tail in the growing embryo. Development of segments-and, later, distinct tissuesrequires a carefully coordinated square dance of gene expression machinery. Proteins coded by special genes called transcription factors call out the steps by binding to DNA to block or encourage expression of specific genes. With tothe-minute timing, transcription factors call other genes into action to produce the proteins that will determine cell fate. Developing organisms express different transcription factors at specific times and locations to coordinate the changes that make some cells head, others tail; one, a neuron, another, muscle.

A transcription factor called Hairy is one of the first activated during segment development in the fruitfly Drosophila melanogaster. Misregulation of Hairy and related factors is associated with cancer and developmental defects across species. Mutations in the gene that codes Hairy lead to overexpression of genes involved in development, but it is not clear whether Hairy normally represses those genes directly, or through intermediaries. To find the answer, Susan Parkhurst and colleagues at the Fred Hutchinson Cancer Research Center in Seattle, Washington, set out to identify Hairy's direct targets.

The researchers found a total of 59 genes bound directly by the Hairy protein in cultured Drosophila cells called Kc cells and in embryos collected at the peak of Hairy expression during Drosophila segmentation. Because they searched approximately half of the expected Drosophila genome, the researchers estimate that they identified roughly half of all Hairy target genes. The list included genes known to act during segmentation,

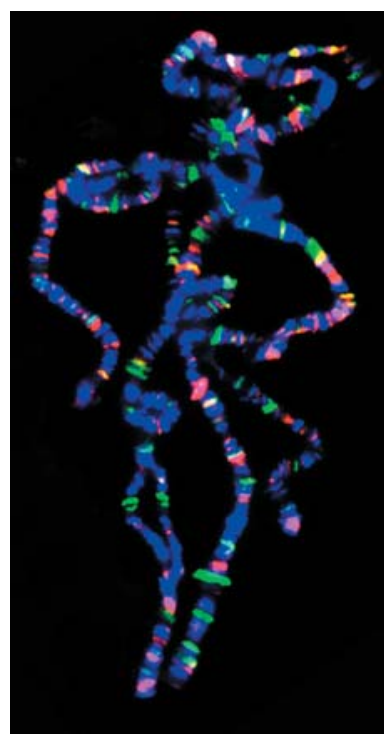

Polytene chromosomes (blue) stained for Hairy (green) and Groucho (red) binding 
as expected, as well as many others with roles in cell division, growth, and shape.

Of the 59 Hairy targets identified, only one appeared in both Kc cells and embryos. The lack of overlap may reflect a difference in developmental stage between the Kc cells, which are thought to be precursors to neurons, and the relatively undifferentiated embryos, and suggests that Hairy's role changes with context, such as the stage of development or tissue type. But Hairy doesn't act alone. Like most transcription factors, Hairy requires assistant proteins, called cofactors, to do its job. The availability of Hairy's known cofactorsGroucho, Drosophila C-terminal binding protein (dCtBP), and Drosophila Sir2
(dSir2) - may help to set the tempo and timing of Hairy's repression of genes.

Groucho was thought to be Hairy's main assistant, but Parkhurst and colleagues found that only one of the identified Hairy target genes bound Groucho in Kc cells. The majority of Hairy targets overlapped with those of dCtBP, most often in combination with dSir2. All of the cofactors also bound to non-Hairy targets, suggesting that they assist other transcription factors as well.

Next, Parkhurst's lab plans to explore the biological functions of the 59 Hairy target genes, to see how they help Hairy coordinate development. Meanwhile, the list of known Drosophila genes has grown 2-fold to include almost all of the expected genome. Repetition of these experiments with the expanded gene set could identify most or all of Hairy's target genes.

The current results suggest that Hairy plays roles in segmentation, cell division, and tissue formation that evolve as an organism develops. Differences in cofactor involvement could help regulate Hairy's repression of genes. This paper demonstrates a powerful technique to explore how developing embryos keep gene expression in step.

Bianchi-Frias D, Orian A, Delrow JJ, Vazquez J, Rosales-Nieves AE, et al. (2004) Hairy transcriptional repression targets and cofactor recruitment in Drosophila. DOI: 10.1371 /journal.pbio.0020178

\section{Remembering Which X Chromosome to Use}

In mammals, males usually have one $X$ and one $Y$ chromosome and females have two $X$ chromosomes. This crucial difference sets the sexes apart, but also creates a problem-female cells have the potential to turn out twice as much X-based gene product as necessary. Males with multiple-X chromosome syndromes face a similar problem. As if to avoid an overdose of X-related proteins, cells in the early embryo inactivate all but one $X$ chromosome. The choice of which X (or Xs) to inactivate is apparently random, but once made, it persists across cell divisions and the specializations that determine a cell's ultimate fate, or type.

A gene called Xist-which resides on the X chromosome-has a central role in $X$ chromosome inactivation. It creates a special RNA molecule that spreads from its point of production down the length of the $X$ chromosome, repressing its genes and inactivating the chromosome. After about one cell cycle, this gene silencing no longer requires Xist RNA. Daughter cells somehow remember which X to keep mute. In this month's PLoS Biology, geneticist Anton Wutz and colleagues at the Research Institute of Molecular Pathology in Vienna show that Xist expression during a critical period very early in embryonic development creates a chromosomal memory, independent of $X$ silencing, that might help maintain $X$ inactivation across cell generations.

The molecular underpinnings of $X$ inactivation seem to center on histones, the protein spools around which DNA coils its length. DNA and histones form complexes called chromatin, which undergoes many structural modifications that have important effects on gene expression. For example, tightly packed chromatin inhibits gene expression in its closely curled segments. Not surprisingly, the inactivated $X$ chromosome is coiled into this dense form, called heterochromatin. In the standing model of $X$ inactivation, the Xist gene mediates alterations to histones (such as the addition of chemical compounds called methyl groups) along the $\mathrm{X}$ chromosome, which result in heterochromatin formation. As this structure is passed on to daughter cells, $X$ silencing is perpetuated.

To explore the molecular changes that mediate $X$ chromosome inactivation, Wutz and colleagues inserted a special Xist gene into the $X$ chromosome of male mouse embryonic stem cells, so they could turn Xist expression "on" and "off" at will. The stem cells represent the earliest, unspecialized cells of a mouse embryo. Since the cells can be induced to differentiate in culture, they provide the opportunity to study the relationship between differentiation and $X$ chromosome inactivation (which would not normally happen at all in these "male" cells). Using this system,

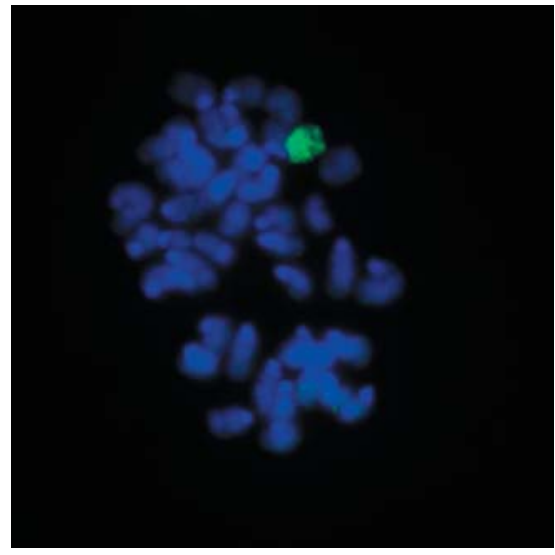

Chromosome-wide histone H3 lysine 27 trimethylation caused by Xist expression the authors have shown previously that Xist must act during a critical window very early during stem cell differentiation-within the first 24 hours. Wutz and colleagues now show that after that window the $X$ chromosome inactivation can still be reversed, but after an additional 24 hours, it cannot. There appears to be a "memory" of Xist action, which leads to the permanent shutting down of the chromosome.

The importance of this observation is that it establishes a new step in the process of $X$ chromosome inactivation-between the action of Xist and the establishment of irreversible silencing. By looking at the kinetics of histone modification, gene silencing, and Xist action, Wutz and colleagues further show that although certain histones are methylated at specific locations during this period in response to Xist, these modifications do not themselves constitute the chromosomal memory. The nature of the memory remains mysterious. Further experiments, perhaps looking at different histone modifications, will be required.Clarification of the events that lead to $X$ inactivation will also improve our knowledge of how changes in the organization and structure of chromosomes can influence the activity of genes.

Kohlmaier A, Savarese F, Lachner M, Martens J, Jenuwein T, et al. (2004) A chromosomal memory triggered by Xist regulates histone methylation in X inactivation. DOI: 10.1371/journal.pbio.0020171 
When It Comes to Frizzled-Mediated Developmental Pathways, Location Matters

The process of morphogenesis has long inspired the wonder and imagination of those who study it. And until the advent of adequate microscopy and lab techniques in the early 19th century, theories based more on imagination-like preformation, which held that sperm harbored fully formed, tiny beings - than observation persisted. But observationally based embryology, it turned out, revealed a notion even more fantastic: the complex higher-order architecture of tissues and organs emerges from a single cell.

Patterns and structures arise largely through cell-to-cell signaling, directed by signaling molecules (ligands) and their receptor targets. These signaling pathways control key developmental processes like cell proliferation and orientation (also called polarity). A relatively small cadre of molecules is enlisted over and over again to initiate an equally limited number of pathways to shape a developing embryo. Though the mechanics and effects of many of these pathways are understood, far less is known about the mechanisms that regulate which pathway is activated. One well-studied family of proteins, called Frizzled (Fz), regulates body symmetry and cell polarity, which, among other things, makes sure the bristles on a fly's wing all point in the same direction. In the fruitfly Drosophila, Fz can activate two distinct developmental pathways: the Wnt/ $\beta$-catenin pathway and the Fz/planar cell polarity (Fz/PCP) pathway. In the Wnt pathway, a Wnt ligand activates the transmembrane Frizzled receptor, which in turn activates the subcellular Disheveled (Dsh) protein, setting off a signaling cascade that ultimately activates genes involved in cell division. The Fz/PCP pathway affects the orientation of wing bristles and the symmetry of the repeating units (ommatidia) in the fly's compound eye.

Previous studies suggest no clear association between a particular ligand-receptor combination and the downstream pathway, begging the question of how similarly structured receptors can signal through a common protein (Dsh) to activate different signaling pathways. As Jun Wu, Thomas Klein, and Marek Mlodzik report in this issue, it's all a matter of being in the right place at the right time.

Since the same Wnt ligand-Fz receptor combinations can produce different results, the researchers reasoned that signaling specificity might depend on the context and cell type. This notion is supported by evidence that Wnt ligands bind at Fz mainly along the basolateral membrane of developing epithelial cells and that Fz hews to the apical membrane of developing wing epithelia during PCP signaling. (The plasma membrane of epithelial cells contains distinct polar domains-the apical and basolateral domains-with distinct properties.) The researchers investigated whether this location bias affects which pathway is activated by focusing on two members of the Fz family: Fz1 and Fz2. Either can activate the Wnt pathway, but only Fz1 is involved in the Fz/PCP pathway. Wu et al. first confirmed that the proteins congregated in distinct subcellular regions of developing wing epithelial cells. Then they looked for sequences or domains in the proteins that might account for their location preferences by creating Fz1/Fz2 hybrids made of various combinations of three different Fz domains. (One was the ligand-binding domain, the second the transmembrane domain, and the third the cytoplasmic "tail.") All the hybrids with a Fz1 tail localized along the apical membrane while those with a Fz2 tail preferred the basolateral membrane, indicating that the tail domain of a receptor controls its location.

The team went on to correlate apical Fz with higher levels of Fz/PCP signaling, based in part on observations that wing hairs point away from areas of $\mathrm{Fz}$ expression, a result associated with PCP signaling. They also showed that increased Fz activity in apical regions results in wing notches and missing bristles - traits associated with reduced Wnt signaling -indicating that apical Fz expression interferes with Wnt/ $\beta$-catenin signaling. That Fz receptors can elicit distinct responses depending on their subcellular location helps explain how so few molecules can juggle so many tasks, including the miraculous feat of building an organism.

Wu J, Klein TJ, Mlodzik M (2004) Subcellular localization of Frizzled receptors, mediated by their cytoplasmic tails, regulates signaling specificity. DOI: 10.1371/journal.pbio.0020158
Harnessing DNA-Based Technology for Drug Discovery

Traditionally, developing small molecules for research or drug treatments has been a painstaking enterprise. Drugs work largely by binding to a target protein and modifying or inhibiting its activity, but discovering the rare compound that hits a particular protein is like, well, finding a needle in a haystack. With a specific protein target identified, scientists typically either gather compounds from nature or synthesize artificial compounds, then test them to see whether they act on the target.

The birth of combinatorial chemistry in the early nineties promised to revolutionize this laborious process by offering a way to synthesize trillions of compounds at a time. Though molecules still had to be evaluated one by one, high-throughput screening technology could manage up to a million molecules a day. Despite these technological advances, few drugs have emerged from combinatorial chemistry approaches, leaving the promise largely unfulfilled.

Another strategy looks to nature as a model. The immune system fights disease and infection by generating billions of antibodies, each primed to recognize a specific pathogen. Antibodies recognize antigens (protein fragments of pathogens) with an exacting specificity that develops through an iterative process. The body first produces a diverse, random collection of antibodies. Every antibody is encoded with a unique DNA blueprint in a B-cell. Antibodies that happen to bind to a pathogen are "selected" to pass on their blueprints: successful binding stimulates cell division, during which blueprints are copied, varied by mutation, and used to create a new generation of antibodies. Specificity is refined over multiple generations.

Over the past fifteen years, biologists have developed techniques to recreate this process in a test tube. Today, it's common practice to "evolve" collections of as many as a quadrillion different proteins or nucleic acids to bind a molecular target. These techniques are called molecular breeding, because like traditional livestock and crop breeding techniques, they combine 


\section{DNA Display}

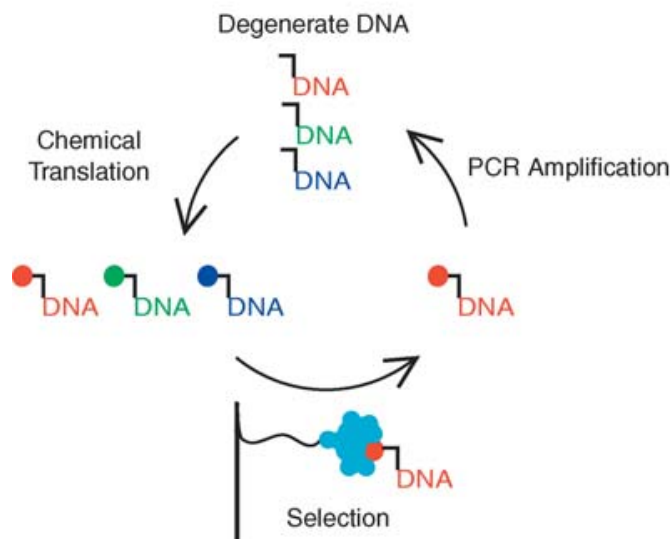

Small-molecule evolution

sets of genotypes over generations to produce a desired phenotype. Molecular breeding has so far been restricted only to applications that involve materials encoded by DNA. Drugs produced by conventional synthetic organic chemistry, for example, cannot be bred.
In a series of three articles in this issue of PLoS Biology, David Halpin et al. describe a strategy that addresses this limitation. By inventing a genetic code that acts as a blueprint for synthetic molecules, the authors show how chemical collections of nonbiological origin can be evolved. In the first article, Halpin et al. present a method for overcoming the technical challenge of using DNA to direct the chemical assembly of molecules. In the second, they demonstrate how the method works and test its efficacy by creating a synthetic library of peptides (protein fragments) and then showing that they can find the "peptide in a haystack" by identifying a molecule known to bind a particular antibody. The third paper shows how the method can support a variety of chemistry applications that could potentially synthesize all sorts of nonbiological "species." Such compounds, the authors point out, can be used for drug discovery or as molecular tools that offer researchers novel ways to disrupt cellular processes and open new windows into cell biology. While medicine has long had to cope with the evolution of drug-resistant pathogens, it may now be possible to fight fire with fire. To learn more about the DNA display method described here, see the primer "Translating DNA into Synthetic Molecules," also in this issue.

Halpin DR, Harbury PB (2004) DNA display I. Sequence-encoded routing of DNA populations. DOI: 10.1371/journal. pbio.0020173

Halpin DR, Harbury PB (2004) DNA display II. Genetic manipulation of combinatorial chemistry libraries for small-molecule evolution. DOI: 10.1371/journal. pbio.0020174

Halpin DR, Lee JA, Wrenn SJ, Harbury PB (2004) DNA display III. Solid-phase organic synthesis on unprotected DNA. DOI: 10.1371/journal.pbio.0020175 